\title{
Simultaneous Single Unit Recording in the Mitral Cell Layer of the Rat Olfactory Bulb under Nasal and Tracheal Breathing
}

\author{
MARÍA DE LA LUZ AYLWIN*, EUGENIA DÍAZ ${ }^{\dagger}$ and PEDRO E. MALDONADO*
}

CENI*, Iniciativa Científica Milenio y Programas de *Fisiología y Biofísica, ${ }^{\dagger}$ Morfología ICBM, Facultad de Medicina, Universidad de Chile, Santiago, Chile.

\begin{abstract}
Odor perception depends on the odorant-evoked changes on Mitral/Tufted cell firing pattern within the olfactory bulb (OB). The OB exhibits a significant "ongoing" or spontaneous activity in the absence of sensory stimulation. We characterized this ongoing activity by simultaneously recording several single neurons in the mitral cell layer (MCL) of anesthetized rats and determined the extent of synchrony and oscillations under nasal and tracheal breathing. We recorded 115 neurons and found no significant differences in the mean firing rates between both breathing conditions. Surprisingly, nearly all single units exhibited a long refractory period averaging $14.4 \mathrm{~ms}$ during nasal respiration that was not different under tracheal breathing. We found a small incidence ( $2 \%$ of neurons) of gamma band oscillations and a low incidence $(8.1 \%)$ of correlated firing between adjacent MCL cells. During nasal respiration, a significant oscillation at the respiratory rate was observed in $12 \%$ of cells that disappeared during tracheal breathing. Thus, in the absence of odorants, MCL cells exhibit a long refractory period, probably reflecting the intrinsic OB network properties. Furthermore, in the absence of sensory stimulation, MCL cell discharge does not oscillate in the gamma band and the respiratory cycle can modulate the firing of these cells.
\end{abstract}

Key terms: Coding, tetrodes, spikes, ongoing activity, synchrony.

\section{INTRODUCTION}

Olfactory perception is the consequence of a combinatorial activation of different glomeruli within the olfactory bulb triggered by the presence of odorants in the nasal epithelia. Olfactory receptor neurons respond to a large variety of odorant molecules with very different chemical structures; these molecules activate different types of olfactory sensory neurons that project to the olfactory bulb, the first stage of sensory processing. Within the OB, olfactory sensory neurons that express the same receptor (Malnic et al., 1999) and have the same molecular receptive properties (Araneda et al., 2000), synapse with the output MCL cells in only two olfactory glomeruli at each OB (Mombaerts et al., 1996). Exposure to odorants triggers a distributed activation of the glomeruli population in the OB (Rubin and Katz, 1999), presumably resulting in a corresponding activity pattern at the MCL cells (Belluscio et al., 2002). This ability to establish spatially distinct activity patterns by different odorants has been proposed to be instrumental in the mechanism of odor coding in the OB (Rubin and Katz, 1999).

However, the olfactory epithelium is not passively exposed to odorants. Vertebrates typically breathe at regular intervals and perform behavioral modulations of the air inflow (sniffing) during natural olfactory tasks. It has been shown that the MCL cell firing rate is modulated by respiration in the presence and absence of odorant stimulation (Macrides and Chorover, 1972;

Corresponding author: María de la Luz Aylwin, Ph.D., Universidad de Chile, Santiago, Chile, Programa de Fisiología y Biofísica, ICBM, Facultad de Medicina, Casilla 70005 Santiago 7, Chile, Tel: (562) 678-6051, Fax: (562) 777-6916. Email: maylwin@med.uchile.cl

Received: March 9, 2004. In Revised Form: December 15, 2004. Accepted: March 2, 2005. 
Chaput and Holley, 1979; Chaput and Holley, 1980; Pager, 1985; Chaput, 1986; Buonviso et al., 1992; Chaput et al., 1992; Sobel and Tank, 1993; Ogawa, 1998). It has been proposed that the origin of this modulation relates to the action of centrifugal pathways on the OB (Davis and Macrides, 1981; Zheng et al., 1987), but the mechanical activation of olfactory sensory cells by the airflow during respiration cannot be completely ruled out. However, it has been postulated that this modulation is solely the result of ambiance odorant stimulation of the olfactory epithelia (Sobel and Tank, 1993). Nevertheless, this respiratory modulation of neuronal firing is present during odorant sampling and may play a role in odorant coding, as is evident in the characteristic sniffing behavior these animals display during odorant discrimination (Kay and Laurent, 1999).

In vertebrates, most of the characterization of $\mathrm{OB}$ neuronal activity has been obtained through the study of MCL cells using single unit recordings, while there is limited information about the activity of the population of neurons recorded simultaneously (but see Buonviso and Chaput, 1990; Kashiwadani et al., 1999). Studies in insects have shown that temporal patterns of activity contribute to the mechanisms underlying olfactory stimulus coding (Laurent et al., 1996) as has been proposed for other sensory systems (Singer, 1999; Gray, 1999). In mammals, however, there are only a few studies that have analyzed the temporal properties of neuronal assemblies of MCL neurons (Buonviso et al., 1996; Kashiwadani et al., 1999). Whatever the coding mechanism underlying olfactory perception, it is superimposed on the ongoing neuronal dynamics.

In this study, we compared the temporal properties of the $\mathrm{OB}$ ongoing activity by simultaneously recording groups of single units in the MCL of anesthetized SpragueDawley rats under nasal and tracheal breathing. Our aim was to characterize, in the absence of odorants, the spike train patterns as well as oscillations and synchrony of groups of adjacent cells in the OB which likely innervate the same or adjacent glomeruli, and thus presumably share similar receptive field properties.

\section{EXPERIMENTAL PROCEDURES}

Animals and surgical preparation: Surgical and experimental techniques were performed in accordance with institutional guidelines and National Institute of Health Guide for the Care and Use of Laboratory Animals (NIH Publications No. 80-23, revised 1996). Male and female SpragueDawley rats weighting 300 to $450 \mathrm{~g}$ were anesthetized with an intraperitoneal injection of ketamine (70 $\mathrm{mg} / \mathrm{kg})$, acepromazine $(7.2 \mathrm{mg} / \mathrm{kg})$ and atropine $(0.01 \mathrm{mg} / \mathrm{kg})$ and supplemented as necessary during the experiment with ketamine $(70 \mathrm{mg} / \mathrm{kg})$, or thiopental $(50 \mathrm{mg} /$ $\mathrm{kg}$ ) IP. We maintained a level of anesthesia in which the nociceptive paw reflex remained completely abolished at all times. We performed small section of the trachea under anesthesia and inserted a y-shaped tube. This tube enabled us to direct the respiratory airflow either through the normal airways (nasal) by occluding the tracheal opening, or directed through the open tube that had low airflow resistance. In the latter case, no measured airflow could be detected through the nasal opening. Body temperature was maintained at $37^{\circ} \mathrm{C}$ degrees with a heating pad. Temperature and electrocardiogram activity was monitored throughout the experiment. The animals were positioned in a stereotaxic apparatus and a craniotomy was performed to expose the dorsal surface of the olfactory bulb. After the experiments, the animals were euthanized with a barbiturate overdose and some of the animals were perfused for histological procedures.

Recording Techniques: Multi-unit activity signals were obtained with customfabricated nichrome tetrodes whose impedance was 1 to $2 \mathrm{MOhms}$ measured at $1 \mathrm{KHz}$. These electrodes can record neurons from a volume with a radius of $65 \mu \mathrm{M}$ (Gray et al., 1995). The multi-unit signals were amplified $(10 \mathrm{~K})$, bandpass filtered 
$(0.1 \mathrm{KHz}-5 \mathrm{KHz})$, and digitized $(25 \mathrm{KHz} /$ channel) using PC custom-designed software. For each of the different data sets, spike separation was achieved by an interactive custom computer program (Gray et al., 1995; Maldonado and Gray, 1996). In this program, selected spike parameters for any two of the four recording channels of the tetrode are displayed in two dimensional scatter plots, revealing a clustering of values. Ellipses are drawn around distinctively clustered data points and the values corresponding to each cluster are assigned a unique color. The clusters can then be iteratively followed and redefined in as many projections as needed to uniquely define a particular single unit. Once a unique cluster is defined, the spike train of each cell was computed by recovering the time stamp of each data point in the cluster. The extracted spike train for each cell was stored with a $1.0 \mathrm{~ms}$ resolution. At each site we recorded the neuronal activity for up to $200 \mathrm{sec}$ in $10-\mathrm{sec}$ duration trials.

Stimulation: Because the experiments were designed to compare the ongoing activity during nasal and tracheal breathing, a constant flow of clean humidified artificial air was directed over the rat's nose and no odorant stimuli were used.

Anatomy: After the recording session, a lesion was made at some selected sites by applying a DC current $(10 \mu \mathrm{A}$ for $10 \mathrm{sec})$ between one of the tetrode's wire and ground. The animal was then overdosed with thiopental $(150 \mathrm{mg} / \mathrm{kg})$ and then perfused with room temperature saline solution followed by $4 \%$ paraformaldehyde in PB. Once the animal was fixed, the brain was removed and stored in $4 \%$ paraformaldehyde at $4{ }^{\circ} \mathrm{C}$. Prior to sectioning, the brain was submerged in $30 \%$ sacarose, sliced $(30 \mu \mathrm{m})$ with a cryostat and stained with cresyl violet.

Data analysis: Auto-correlation, crosscorrelation and inter-spike interval histograms (ISI) were computed using MATLAB and LabWindows software. To quantify neuronal rhythmicity, we calculated the power spectrum of each autocorrelogram $(\mathrm{ACH})( \pm 128 \mathrm{~ms})$ and extracted the frequency and amplitude of the peak value in the frequency range of 20$100 \mathrm{~Hz}$. The statistical significance of the spectral peaks was estimated using a Monte Carlo simulation. For this procedure, we generated an equivalent pseudo random spike train for each window of data collected on each trial using a random sample from a uniform distribution and a refractory period similar to that obtained for the test ACH (Friedman-Hill et al., 2000). The ACH and corresponding power spectrum were computed for each simulated data set and the peak value in the spectrum between 20-100 Hz was extracted. The simulation was repeated 500 times and any experimental value was considered significant if it was greater than the $99 \%$ of simulated value (cutoff value).

To quantify neuronal synchrony in each cross-correlogram $(\mathrm{CCH})$, we compared the central area in each correlogram to a computed equivalent pseudo random spike trains $\mathrm{CCH}$ with identical spike count, mean firing rate and duration, to the experimental data. As with the autocorrelation analysis, both simulations were repeated 500 times for each histogram. See details in (Maldonado et al., 2000). Significant correlograms were $\mathrm{CCH}$ with central peaks above $99 \%$ of those seen for simulated data. The refractory period was calculated directly from the ACHs and was defined as the time between the center of the autocorrelogram and the time to reach half amplitude of the second peak of the autocorrelogram. Statistical significance of differences between means was estimated using the paired t-test.

\section{RESULTS}

We recorded 115 neurons from 51 sites in 10 rats, ranging from 1 to 6 cells per site and an average of 3 cells per site. The recordings were made in the dorsal and ventral layers of the anterior $2 / 3$ of the OB. Tetrode penetrations were achieved with a micro manipulator, and the MCL was located by its large-amplitude extracellular activity. An 
example of a recording in the MCL is shown in Figure 1A. Extracellularly-recorded action potentials of neurons located near the tip of the electrode display different amplitudes depending on their spatial distribution with respect to the four wires of the tetrode. There is a clear difference in amplitude in the spikes recorded with each wire. Fifty overlapping waveforms (1.6 $\mathrm{ms}$ in duration) for each of 6 identified single units recorded

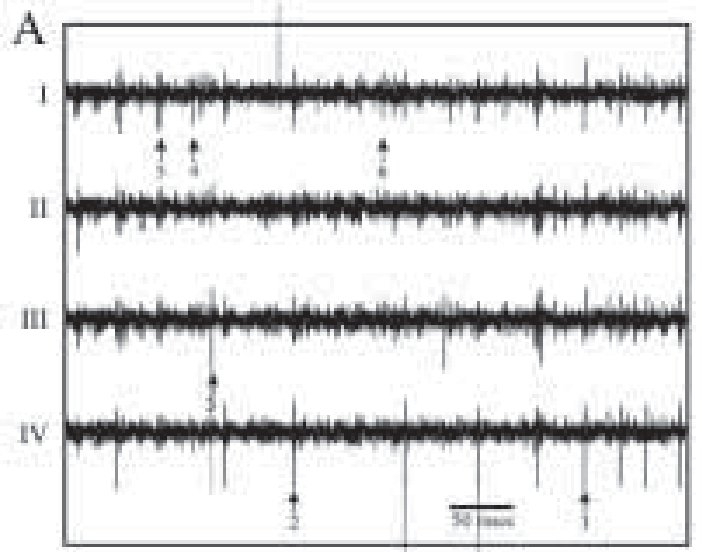

B

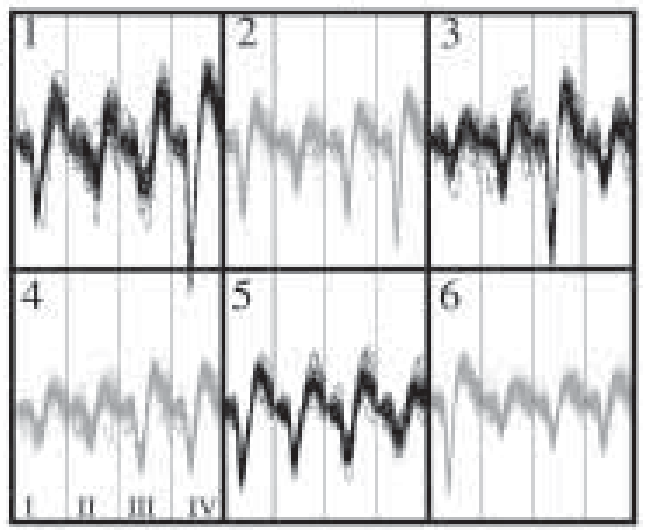

Figure 1. Example of a tetrode recording in the MCL. (A) Traces showing the data recorded with the 4 tetrode wires. Extracellular action potentials recorded from neurons located near the tip of the electrode display different amplitudes depending on their spatial distribution. (B) Traces of 50 overlapping unaveraged waveforms (1.6 $\mathrm{ms}$ in duration) for each of the 6 sorted single units recorded on each channel (I, II, III, and IV) of the tetrode. Note the clear differences in spike amplitude on adjacent channels and how the ratios of these differences vary from cell to cell. Waveforms are captured from an estimated tissue volume of $65 \mu \mathrm{m}$ radius. on each channel (I, II, III and IV) of the same recording are shown in Figure 1B. The properties of the spike waveforms were used to sort all single units from each tetrode recording as described previously (Gray et al., 1995). To confirm the actual recording site in the OB we performed an electrolytic lesion in 3 animals and the site was located after histological staining. All the sites recovered showed the lesion to be located at or around the MCL (data not shown).

Because normal breathing and sniffing has been shown to modulate firing in MCL neurons, we compared the mean firing rate for all cells under nasal and tracheal breathing. The histogram of the mean firing rate for all 115 cells recorded under nasal and tracheal breathing is shown in Figure 2. The average mean firing rate for nasal breathing was $10.3 \pm 6.9 \mathrm{~Hz}$ (mean $\pm \mathrm{SD}$ ) and during tracheal breathing was $10.1 \pm$ $6.5 \mathrm{~Hz}$ (mean $\pm \mathrm{SD})$. We found no statistically significant difference between the means ( $t$-test). Furthermore, even though the distribution of mean firing rate for tracheal breathing was less uniform than the one obtained during nasal breathing, this difference was not statistically significant (Kolmogorov-Smirnov test)

Because the respiratory cycle does not modify the mean firing rates of MCL neurons, we looked for subtler modulations of the temporal patterns of activity between nasal and tracheal breathing. We calculated short lag (128 ms) autocorrelograms (ACHs) for all the cells under nasal and tracheal conditions. Figure 3 shows an example of an $\mathrm{ACH}$ for 4 cells recorded simultaneously under nasal and tracheal breathing. We examined the $115 \mathrm{ACH}$ and concluded that most of the neurons recorded had an evident and characteristic long silent period around the central spike, which we refer to hereafter as the "refractory period," with a duration ranging from 5 to $25 \mathrm{~ms}$. An example of this refractory period in 4 different cells recorded simultaneously by the same tetrode is shown in Figure 3. This long refractory period was also present in the interspike interval histograms. An example of the ISI histogram for the same 4 simultaneously recorded cells shown in figure 3 is shown in Figure 4. There is a clear silent period in all 4 cells 
similar to the one obtained from the ACHs. We found that spike trains with little or no refractory period were uncommon, and more distinctly, an indication of recordings where an unequivocal single unit isolation was not feasible. Therefore, the data with a short o no refractory period were not included in the analysis.

Additionally, we compared the distributions of refractory periods for nasal and tracheal breathing conditions (Fig. 5).

Most cells have a rather long refractory period, with only 19 and 18 cells of the 115 neurons having periods of less than $10 \mathrm{~ms}$ during nasal and tracheal breathing respectively. The mean duration of the refractory period was $14.4 \pm 5.2 \mathrm{~ms}$ (mean $\pm \mathrm{SD}$ ) during nasal breathing; this duration was not significantly different under tracheal breathing conditions (14.9 \pm 5.2 ms, mean $\pm \mathrm{SD}$ ).

Previous reports have emphasized the importance of oscillatory activity in the $\mathrm{OB}$, primarily in the gamma band, and its role in olfactory coding (Gray and Skinner, 1988; Laurent, 1999). In order to estimate the extent of oscillatory ongoing activity in MCL neurons, we examined

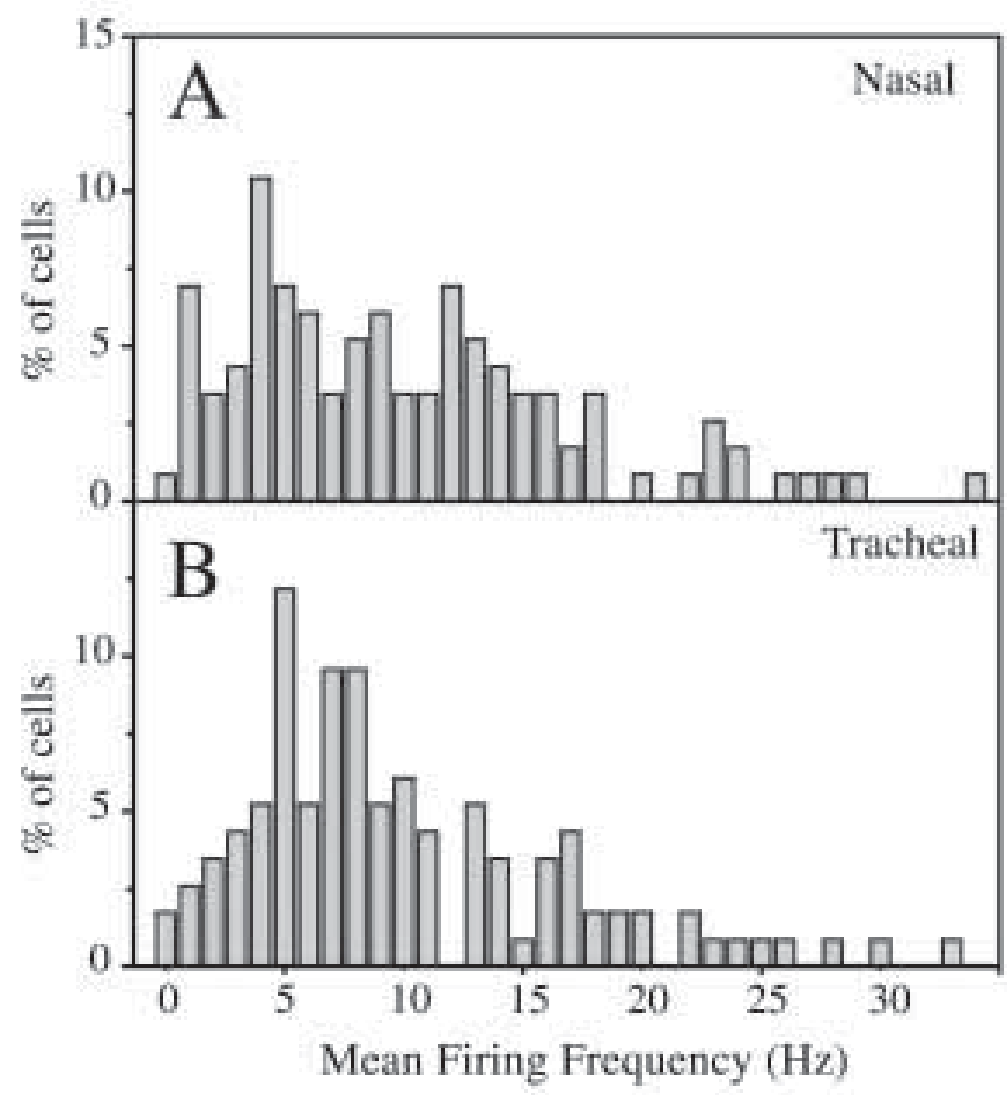

Figure 2. Mean firing frequency distributions of MCL neurons are similar during nasal (A) and tracheal (B) breathing. For each cell, we computed the mean firing rate for 20 trials of $10 \mathrm{~s}$ duration each. This figure shows the frequency distributions for the 115 neurons recorded. Mean firing frequency was $10.3 \pm 6.9$ $\mathrm{Hz}$ and $10.1 \pm 6.5 \mathrm{~Hz}$ for nasal and tracheal breathing respectively. A paired $t$-test showed no significant difference between means obtained in the two breathing conditions. A Kolmogorov-Smirnoff test shows no significant difference between firing rate distributions. 


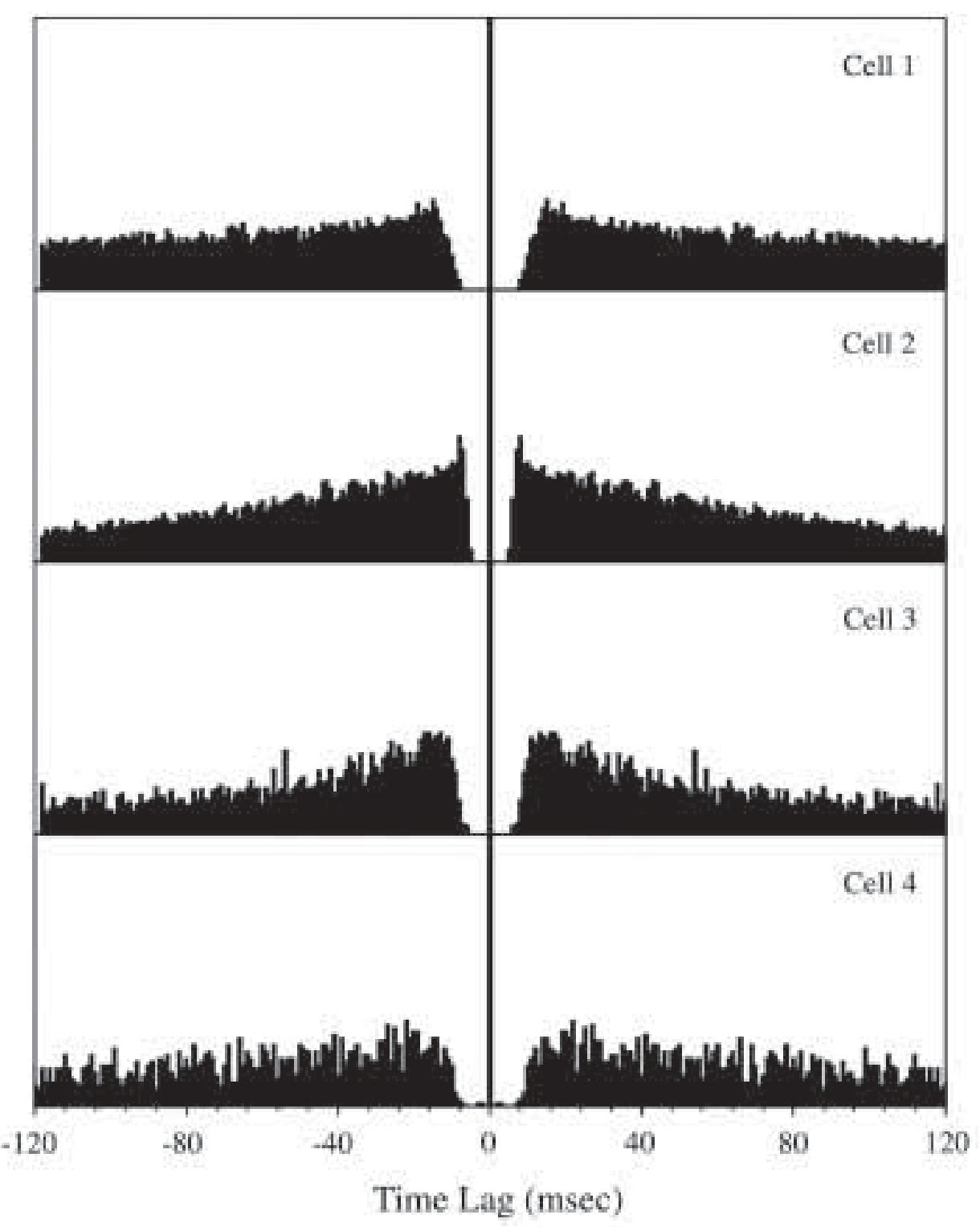

Figure 3. Autocorrelograms (ACHs) reveal a characteristic long refractory period and show no oscillatory firing of MCL neurons. This figure shows an example of the $\mathrm{ACH}$ obtained for four simultaneously recorded cells in one tetrode during nasal breathing. From the ACH we calculated the refractory period as the time between the center of the $\mathrm{ACH}$ and the time to reach half amplitude of the secondary peak. These cells located in close proximity show different refractory periods. The oscillatory activity was assessed by computing the power spectrum of the $\mathrm{ACH}$ and then comparing the peak amplitude with peak distribution of power spectrum of simulated spikes trains with similar statistics. None of the cells shown in this figure have a significant oscillatory firing. Neither the refractory period nor the oscillatory behavior changed when airflow was switched from nasal to tracheal breathing. 


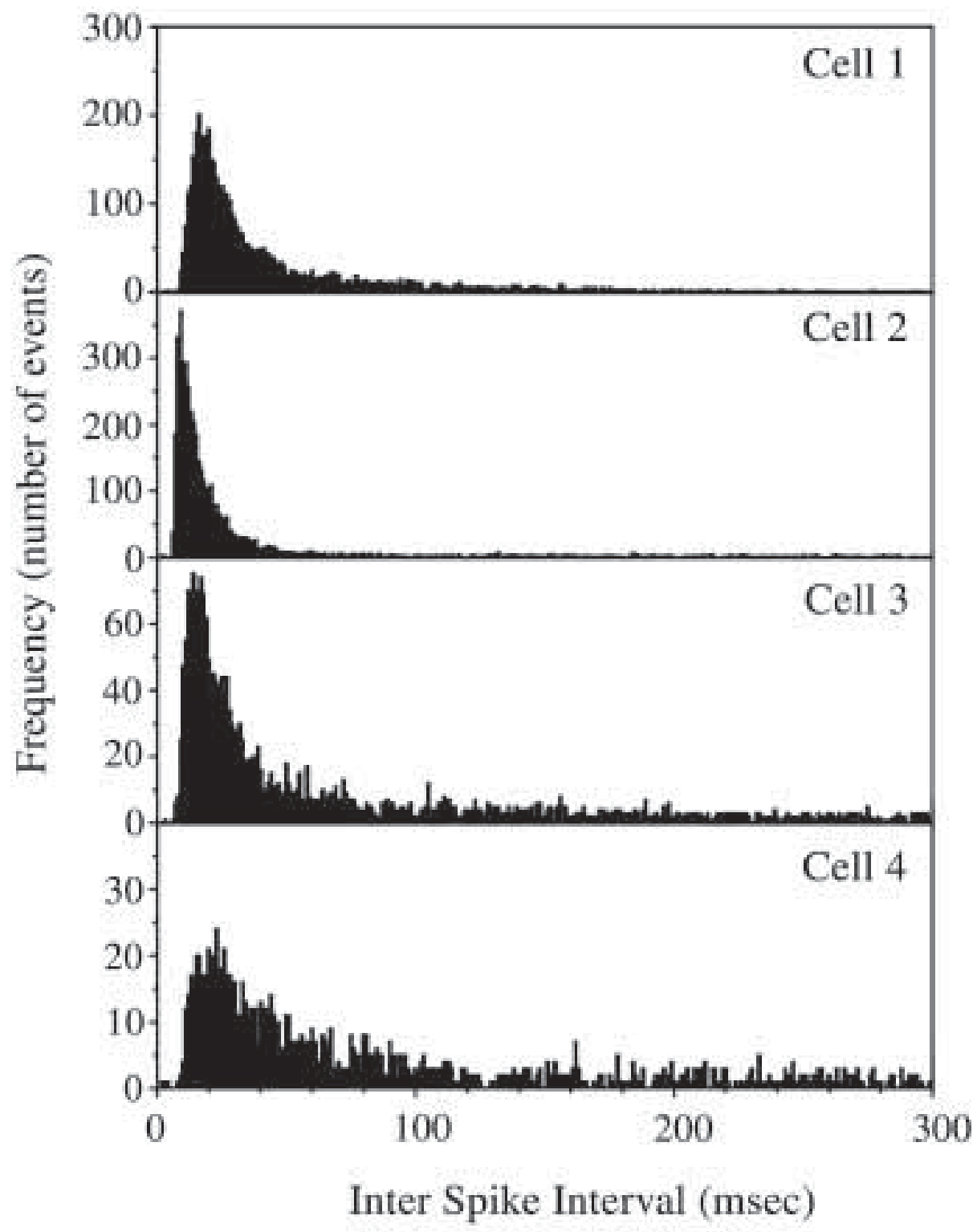

Figure 4. Interspike interval histograms from the same $4 \mathrm{MCL}$ cells recorded simultaneously shown in Figure 3. These distributions are consistent with the refractory period obtained with the ACHs. In these plots, a noticeable difference in peak timing can be observed. 


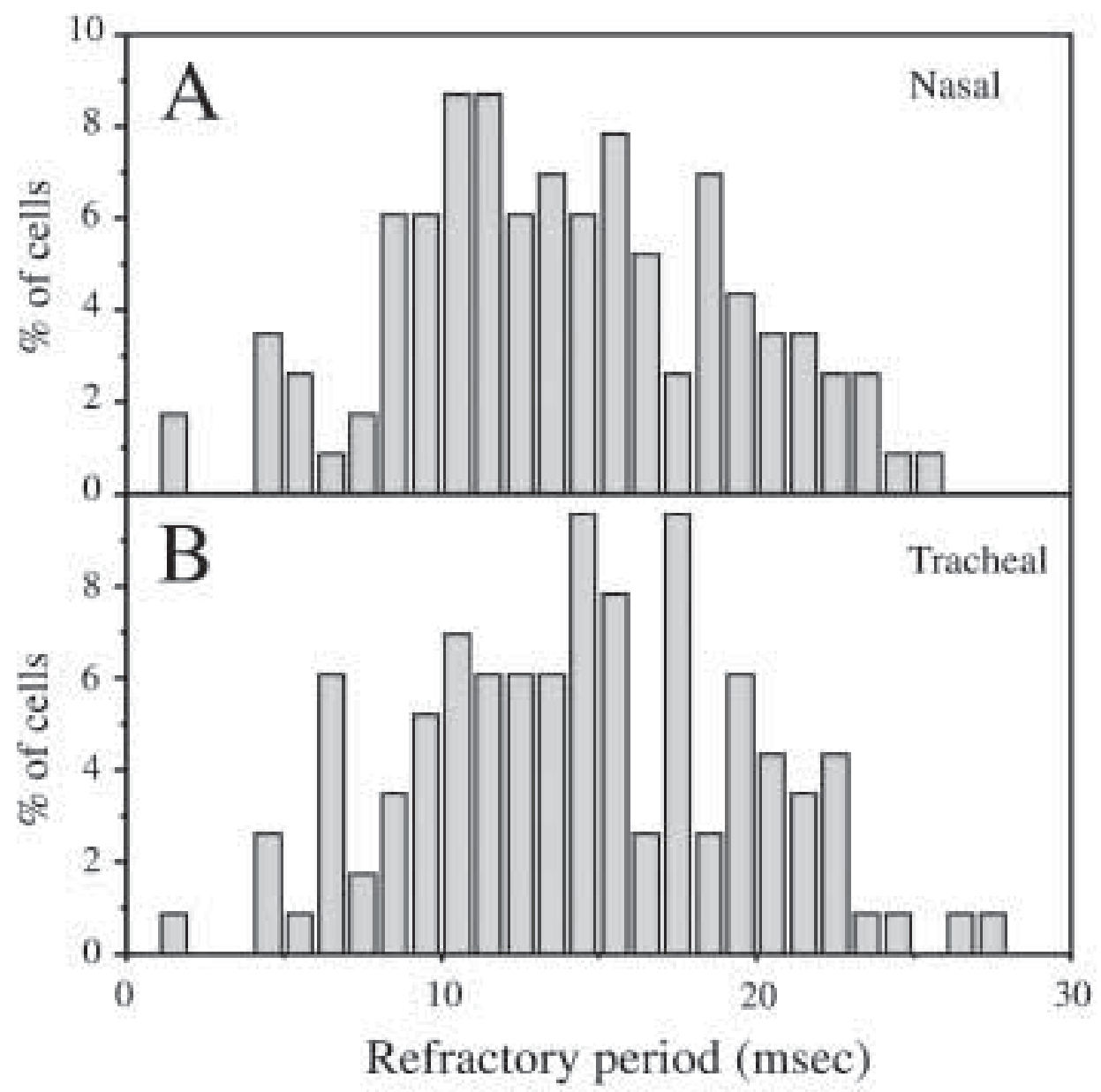

Figure 5. The distributions of refractory periods of MCL neurons is similar during nasal (A) and tracheal (B) breathing $(\mathrm{n}=115)$. The mean refractory periods were $14.4 \pm 5.2 \mathrm{~ms}$ and $14.9 \pm 5.2 \mathrm{~ms}$, for nasal and tracheal breathing respectively. A paired $t$-test of the data found no significant difference between the means corresponding to the two breathing conditions.

the gamma frequency range $(35-100 \mathrm{~Hz}$, 28-10 ms period) oscillation of the single unit activity by computing the power spectrum of each cell ACHs during nasal and tracheal breathing. We found that only 2 out of 115 auto-correlograms showed a significant oscillatory activity during nasal breathing and only one of these cells remained oscillatory during tracheal breathing. Furthermore, a more careful examination of the ACHs revealed that most of the power arises from the presence of a secondary peak after the refractory period, which we take as evidence of spike bursting (see Fig. 3). In summary, we found little evidence of rhythmic firing in the single unit activity in the absence of odorant stimulation either during nasal or tracheal breathing.

There is evidence that the respiratory cycle induces a temporal pattern of discharge in the MCL cells (Macrides and Chorover, 1972; Chaput and Holley, 1979; Chaput and Holley, 1980; Pager, 1985; Chaput, 1986; Buonviso et al., 1992; Sobel and Tank, 1993; Chaput et al., 1992; Sobel and Tank, 1993; Ogawa, 1998) and that this modulation can be 
present even in the absence of airflow through the nasal route (Ravel et al., 1987). In order to examine the incidence of respiratory modulation we computed a longer time lag ACHs (2 s) of the MCL cells for the nasal and tracheal breathing conditions. We found that 12 out of 115 cells exhibited clear oscillatory behavior in the firing pattern whose oscillation frequency was in the respiratory range (1$5 \mathrm{~Hz}$ ) during nasal breathing and that this oscillation completely disappeared after the switch to tracheal breathing (Fig. 6). However, when we compared the modulation induced by respiration in different cells recorded simultaneously in the same recording site, we found that some but not all cells exhibited this respiratory modulation during nasal respiration. The example in Figure 6 shows the $\mathrm{ACHs}$ for 3 cells recorded simultaneously in the same site under nasal and tracheal breathing. During nasal respiration there is a noticeable modulation in cells 1 and 2 , but not in cell 3. However, this modulation disappears in all cells during tracheal breathing.

To determine the incidence of the correlated discharge of adjacent MCL cells in the absence of odorant stimulation we computed the cross-correlograms (CCHs) for 247 cell pairs recorded under nasal and tracheal conditions. An example of $\mathrm{CCHs}$ for 3 cell pairs is shown in Figure 7 where there is a significant correlation only in pair 1. In summary, we found that $20(8.1 \%$ nasal) and 11 (4.4\% tracheal) cell pairs
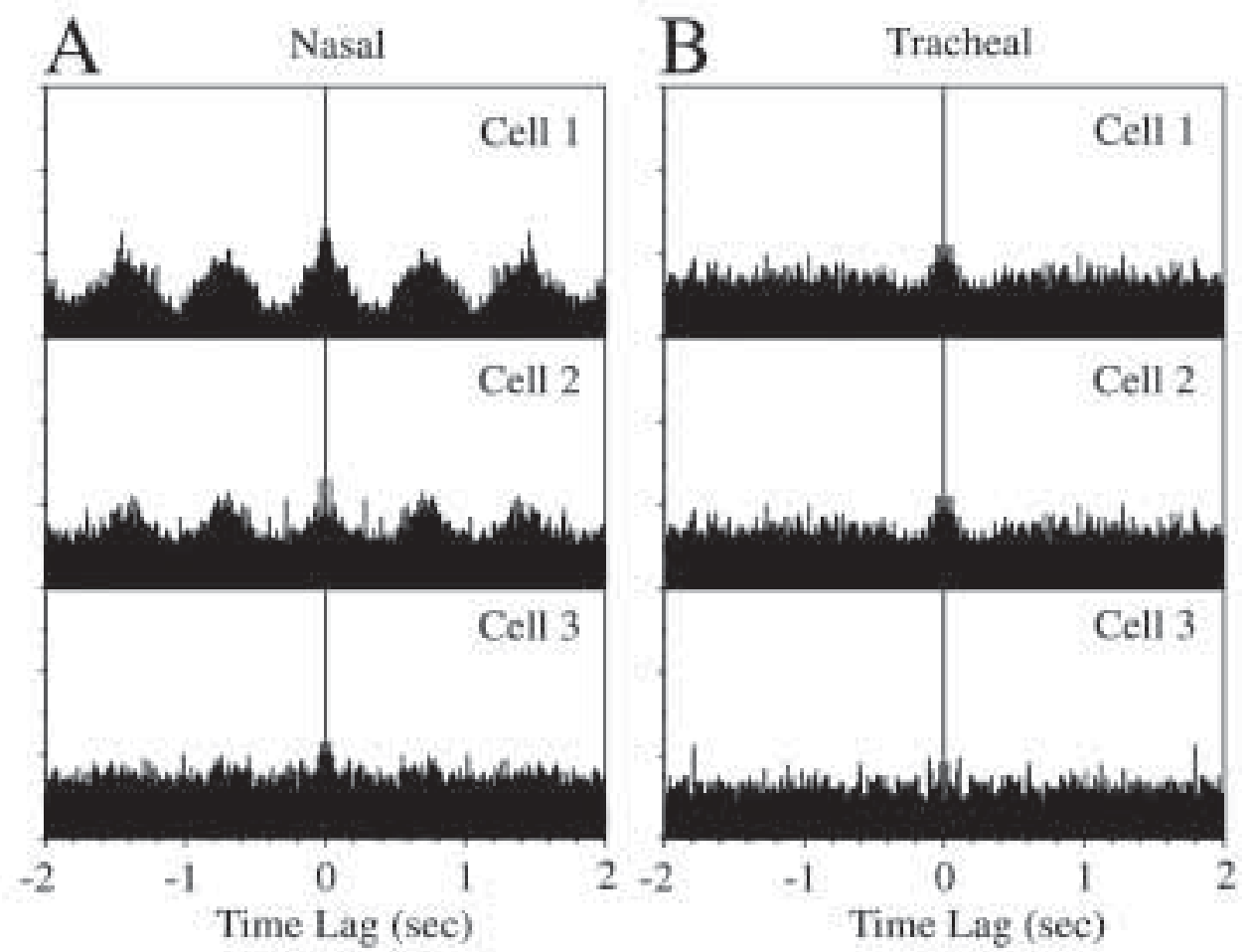

Figure 6. Single unit recordings exhibit a slow modulation of the frequency of firing during nasal breathing but not during tracheal breathing. Here we show a long time lag (2 s) ACHs of single unit activity for 3 cells recorded simultaneously in the same site (A). Note the clear modulation in the $1-3 \mathrm{~Hz}$ range in cells 1 and 3 , but not in cell 2 . This rhythm was always absent in the same cells recorded during tracheal breathing (B). 


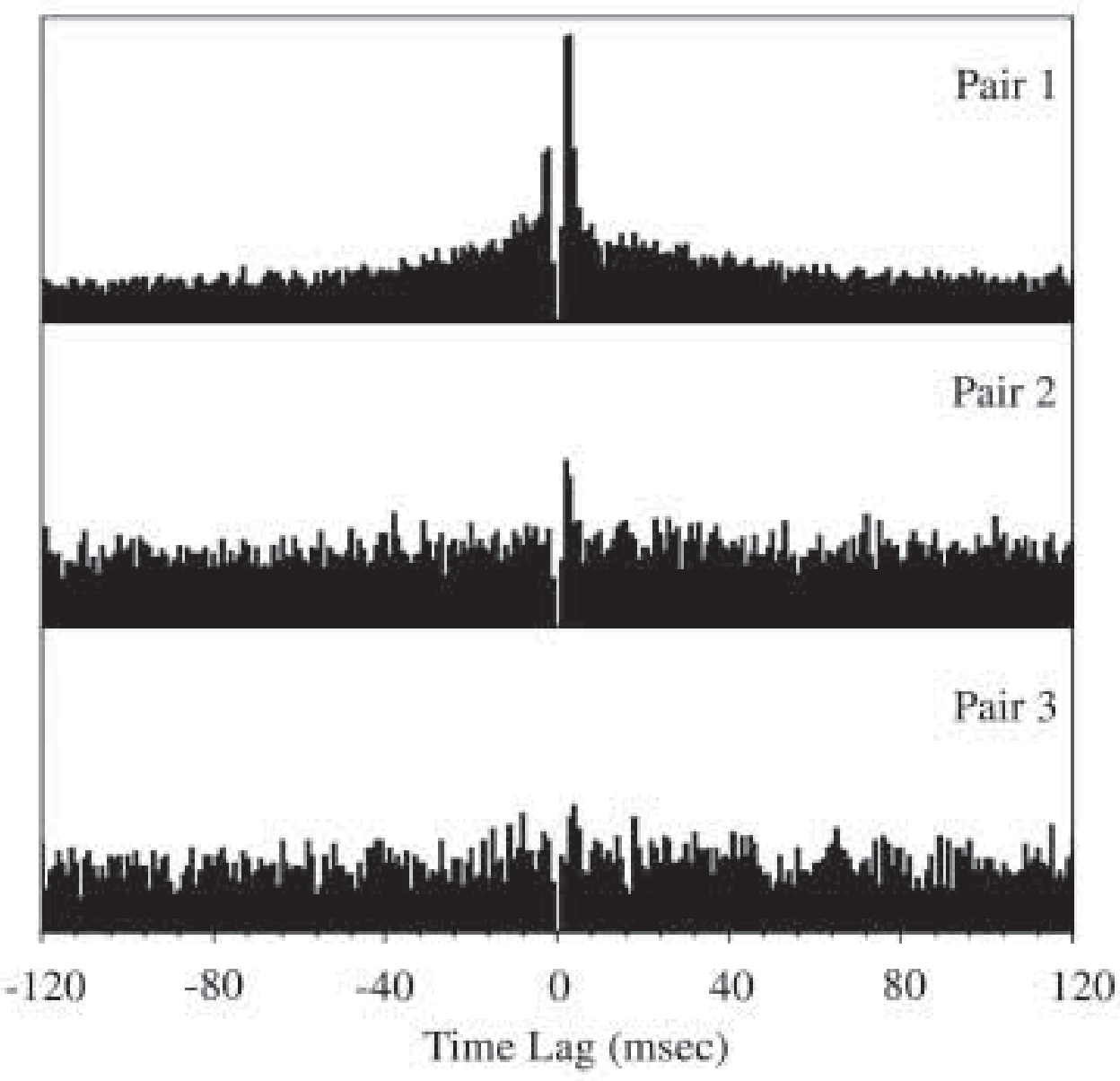

Figure 7. Few MCL neurons synchronize their firing in the millisecond time scale. An example of 3 cell pairs recorded simultaneously for the same tetrode is shown. Of these cell pairs, only pair 1 reached a significant level of correlation. We computed the cross-correlogram for 247 cell pairs and we found that 20 (nasal) and 11 (tracheal) cell pairs showed significant CCHs. The significance level was assessed using a Montecarlo simulation of a Poisson distributed spike train of cells with the same number of spikes (see text for details).

showed significant cross-correlograms. The difference in correlation between nasal and tracheal breathing appears to be independent of the respiratory condition because of the 20 cell pairs that showed significant correlation during nasal breathing only 5 pairs were also significantly correlated after switching to tracheal breathing. Furthermore, the incidence and magnitude of the correlation was not significantly different between nasal and tracheal breathing.
This work takes advantage of the properties of the tetrode that enable the reliable simultaneous recording of single units located in a volume of tissue with an approximate $65 \mu \mathrm{m}$ radius. Using this technique, we were able to characterize the ongoing activity of well-isolated adjacent neurons in the $\mathrm{OB}$ and determine the incidence of correlated firing between neighboring cells in the MCL. We typically recorded extracellular activity only in a small layer of approximately 100-150 $\mu \mathrm{m}$ 
located around $400 \mu \mathrm{m}$ deep corresponding to the dorsal MCL and again in much deeper penetration corresponding to the ventral MCL. This recording pattern agrees with previous work describing the difficulties of recording extracellular single units from the granule cell layer due to the small size and high density of cells in this layer (Kay and Laurent, 1999). We also noted a considerable increment in the oscillations of the local field potential as the electrode enters the granule cell layer (data not shown). Further confirmation of the recording sites came from the histological analysis of lesions performed in the MCL after the recordings.

Previous reports of respiratory modulation of neuronal activity in the OB, led us to expect we would find a difference in the firing rates between the two breathing conditions. We found no significant difference in the mean firing rates between these two conditions, indicating that the respiratory cycle does not modify the overall firing rate of the MCL cells. However, with $\mathrm{ACH}$ analysis using long time lags we found that many cells are indeed modulated by the respiratory cycle, not through modifications in the average firing rate, but by introducing a distinctive temporal modulation that includes an oscillatory pattern of discharge that follows the respiratory cycle. Our results agree with previous studies that demonstrated a significant modulation of firing rate in naturally- or artificially-breathing animals (Onoda and Mori, 1980; Pager, 1985; Ravel et al., 1987; Ravel and Pager, 1990 Sobel and Tank, 1993), but differ from others in that we found no cells that maintain respiratory modulation while the airflow was diverted through the tracheal tube (Ravel et al., 1987). Different explanations have been given to the respiratory modulation of the $\mathrm{OB}$ firing: a peripheral origin based on the mechanical stimulation of the epithelia by the nasal airflow through the nasal cavity, a central origin through the centrifugal connections to the OB (Ravel et al., 1987) and an ambience odorant stimulation of the olfactory epithelia. Our results are consistent with the mechanical or ambience stimulation of the epithelia because the modulation disappears when the airflow is directed through the trachea, despite the fact that the centrifugal connections are intact. Furthermore, we argue that our results could not be the effect of ambience odorant stimulation of the epithelia as clean air was constantly blown over the rat's nose.

A significant amount of work has suggested that the OB oscillatory activity participates in the process of olfactory coding and discrimination (Adrian, 1950; Bressler, 1987; Gray and Skinner, 1988). This oscillatory activity has been primarily observed in EEG and LFP signals and is typically triggered by odorant exposure (Adrian, 1950, Bressler, 1987, Buonviso et al., 2003). However, oscillatory activity in the gamma band has been observed in the absence of odorant stimulation (Martin et al., 2004). We examined the possibility of an oscillatory discharge of MCL cells in the gamma band $(35-100 \mathrm{~Hz})$ and compared its incidence under nasal and tracheal breathing. We found very few significant auto-correlograms in either condition, indicating that there was no oscillatory behavior of the MCL cells under nasal or tracheal breathing in the absence of odorants. However we also have indications that the local field potential signals recorded with the same tetrodes do show a clear gamma band power (Egaña et al., 2002) indicating that the spontaneous discharge does not follow the LFP oscillations in this range. An alternative possibility is that these cells do fire in synchrony with a background LFP (Kashiwadani et al., 1999), but they do so intermittently with low probability, as has been described in the insect antennal lobe (Laurent, 1997; Friedrich and Laurent, 2001). In any event, the relationship between the MCL neurons to the LFP oscillatory activity needs to be further examined.

One of the most interesting features of the ongoing activity of MCL cells under both nasal and tracheal breathing was the conspicuous presence of a long refractory period recorded in almost all of the neurons. The computed inter-spike interval 
histograms confirm this long refractory period. The time inverse of the refractory period is approximately $70 \mathrm{~Hz}$, which is very close to the LFP oscillation frequencies reported in MCL and granule cell layer (Bressler, 1987; Egaña et al., 2002; Buonviso et al., 2003; Neville and Haberly, 2003), yet we found that the single unit discharge from MCL cells did not oscillate. One way to reconcile these observations involves the possibility that neuronal activity is locked to the background oscillation, as recent evidence shows (Kashiwadani et al., 1999; Egaña et al., 2002), but fire only one or two action potentials before being strongly inhibited. To our knowledge, this extended refractory period appears to be unique to MCL neurons and is not found in cortex or thalamic structures, even when these cells clearly oscillate in the gamma band frequency. We found no previous reports of this temporal feature of MCL cells in the mammalian olfactory bulb. However, in the moth's antennal lobe discharge patterns of neurons, only one out of the three cell types described showed a similar long refractory period; the other two exhibited a much shorter refractory period (Christensen et al., 2000). The most likely explanation for this discrepancy is that it could be due to differences in the firing properties among species, or much less likely, to the spike sorting methods.

This long refractory period is probably the result of the reciprocal synaptic connectivity between the MCL cells and the granule cells in the $\mathrm{OB}$. The activation of the reciprocal synapse is temporally consistent with an average $14.4 \mathrm{~ms}$ delay and could at least in part contribute to an oscillatory activity in the gamma range (40 to $100 \mathrm{~Hz}$ ). However, reports have shown that there are subthreshold oscillations in the voltage membrane of mitral cells which vary with the MCL cell depolarization ranging from $100 \mathrm{~ms}$ at $-65 \mathrm{mV}$ to $25 \mathrm{~ms}$ at $-59 \mathrm{mV}$ in a slice preparation These intrinsic oscillations could also give rise to the gamma oscillations recorded in the LFP and EEG (Desmaisons et al., 1999). However, the maximum frequency of oscillations obtained in the slice preparation was $40 \mathrm{~Hz}$, and in the whole animal this could reach $70 \mathrm{~Hz}$ (Buonviso et al., 2003; Neville and Haberly, 2003). Further studies need to be done to determine the mechanisms involved in this refractory period and whether this is the result of reciprocal network dynamics, the intrinsic properties of $\mathrm{OB}$ neurons, or both.

The glomerulus, and all the olfactory receptor neurons and MCL cells that converge and synapse within it, has been proposed as a functional unit in the OB. Consistent with this hypothesis, the activity evoked by odorants has been found to be correlated in cells that are close together and possibly connected to the same glomerulus, compared with the ones that are located farther away (Buonviso and Chaput, 1990; Buonviso et al., 1992). Our results of cross correlation analysis of MCL cells indicate that there is little or no synchronous activity during nasal or tracheal breathing in the absence of odorant stimulation. These results are consistent with previous studies done in rabbit and fish showing that nearby cells exhibit synchronous activity only in the presence of odorants (Nikonov et al., 2002) and that in the absence of odorant stimulation, a very small percentage of cells exhibit synchronized firing in the millisecond time scale (Kashiwadani et al., 1999).

Taken together, our results demonstrate that the dynamics of firing of MCL neurons in the $\mathrm{OB}$ of rats freely breathing through the nose or a tracheal tube are similar in the absence of odorants, differing only in a slow modulation of the frequency of firing produced by the air flow through the nasal cavities during natural respiration. We have also shown that MCL neurons exhibit unusual firing properties characterized by a long refractory period that is compatible with the oscillations described in the LFP, which presumably arise from the OB local intrinsic or network interactions.

\section{ACKNOWLEDGMENTS}

We thank Cecilia Babul and Isaac Oporto for providing animal care and laboratory assistance. Supported by FONDECYT 
Grants 1000901, 1010811, and Iniciativa Científica Milenio ICM P01-007F.

\section{REFERENCES}

ADRIAN ED (1950) The electrical activity of the mammalian olfactory bulb. 2:377-388

ARANEDA RC, KINI AD, FIRESTEIN S (2000) The molecular receptive range of an odorant receptor. Nat Neurosci 3: 1248-1255

BELLUSCIO L, LODOVICHI C, FEINSTEIN P, MOMBAERTS P, KATZ LC (2002) Odorant receptors instruct functional circuitry in the mouse olfactory bulb. Nature 419: 296-300

BRESSLER SL (1987) Relation of olfactory bulb and cortex. I. Spatial variation of bulbocortical interdependence. Brain Res 409: 285-293

BUONVISO N, CHAPUT MA (1990) Response similarity to odors in olfactory bulb output cells presumed to be connected to the same glomerulus: electrophysiological study using simultaneous single-unit recordings. J Neurophysiol 63: 447-454

BUONVISO N, CHAPUT MA, BERTHOMMIER F (1992) Temporal pattern analyses in pairs of neighboring mitral cells. J Neurophysiol 68: 417-424

BUONVISO N, CHAPUT MA, BERTHOMMIER F (1996) Similarity of granular-induced inhibitory periods in pairs of neighboring mitral/tufted cells. J Neurophysiol 76: 2393-2401

BUONVISO $\mathrm{N}$, AMAT $\mathrm{C}$, LITAUDON P, ROUX $\mathrm{S}$, ROYET JP, FARGET V, SICARD G (2003) Rhythm sequence through the olfactory bulb layers during the time window of a respiratory cycle. Euro $\mathrm{J}$ Neurosci 17: 1811-1819

CHAPUT MA (1986) Respiratory-phase-related coding of olfactory information in the olfactory bulb of awake freely-breathing rabbits. Physiol Behav 36: 319-324

CHAPUT M, HOLLEY A (1979) Spontaneous activity of olfactory bulb neurons in awake rabbits, with some observations on the effects of pentobarbital anaesthesia. J Physiol (Paris) 75: 939-948

CHAPUT M, HOLLEY A (1980) Single unit responses of olfactory bulb neurones to odour presentation in awake rabbits. J Physiol (Paris) 76: 551-558

CHAPUT MA, BUONVISO N, BERTHOMMIER $F$ (1992) Temporal Patterns in Spontaneous and OdorEvoked Mitral Cell Discharges Recorded in Anesthetized Freely Breathing Animals. Euro J Neurosci 4: 813-822

CHRISTENSEN TA, PAWLOWSKI VM, LEI H, HILDEBRAND JG (2000) Multi-unit recordings reveal context-dependent modulation of synchrony in odorspecific neural ensembles. Nat Neurosci 3: 927-931

DAVIS B.J., MACRIDES F. (1981) The organization of centrifugal projections from the anterior olfactory nucleus, ventral hippocampal rudiment, and piriform cortex to the main olfactory bulb in the hamster: an autoradiographic study. J Comp Neurol 203: 475-493

DESMAISONS D, VINCENT JD, LLEDO PM (1999) Control of action potential timing by intrinsic subthreshold oscillations in olfactory bulb output neurons. J Neurosci 19: 10727-10737

EGAÑA JI, AYLWIN ML, MALDONADO PE (2002) Multiple single unit recordings and local field potentials (LFPs) recorded with tetrodes in the olfactory bulb (OB) of anesthetized rats. Program No 66022002 Abstract Viewer/Itinerary Planner
Washington, DC: Soc for Neurosci, 2002 CD-ROM

FRIEDMAN-HILL S, MALDONADO PE, GRAY CM (2000) Dynamics of striate cortical activity in the alert macaque: I. Incidence and stimulus-dependence of gamma-band neuronal oscillations. Cereb Cortex 10: $1105-1116$

FRIEDRICH RW, LAURENT G (2001) Dynamic optimization of odor representations by slow temporal patterning of mitral cell activity. Sci 291: 889-894

GRAY CM (1999) The temporal correlation hypothesis of visual feature integration: still alive and well. Neuron 24: $31-25$

GRAY CM, SKINNER JE (1988) Centrifugal regulation of neuronal activity in the olfactory bulb of the waking rabbit as revealed by reversible cryogenic blockade. Exp Brain Res 69: 378-386

GRAY CM, MALDONADO PE, WILSON M, MCNAUGHTON B (1995) Tetrodes markedly improve the reliability and yield of multiple single- unit isolation from multi-unit recordings in cat striate cortex. J Neurosci Meth 63: 43-54

KASHIWADANI H, SASAKI YF, UCHIDA N, MORI K (1999) Synchronized oscillatory discharges of mitral/ tufted cells with different molecular receptive ranges in the rabbit olfactory bulb. J Neurophysiol 82: 17861792

KAY LM, LAURENT G (1999) Odor- and contextdependent modulation of mitral cell activity in behaving rats. Nat Neurosci 2: 1003-1009

LAURENT G (1997) Olfactory processing: maps, time and codes. Curr Opin Neurobiol 7: 547-553

LAURENT G (1999) A systems perspective on early olfactory coding. Sci 286: 723-728

LAURENT G, WEHR M, DAVIDOWITZ H (1996) Temporal representations of odors in an olfactory network. J Neurosci 16: 3837-3847

MACRIDES F, CHOROVER SL (1972) Olfactory bulb units: activity correlated with inhalation cycles and odor quality. Sci 175: 84-87

MALDONADO PE, GRAY CM (1996) Heterogeneity in local distributions of orientation-selective neurons in the cat primary visual cortex. Vis Neurosci 13: 509-516

MALDONADO PE, FRIEDMAN-HILL S, GRAY CM (2000) Dynamics of striate cortical activity in the alert macaque: II. Fast time scale synchronization. Cereb Cortex 10: 1117-1131

MALNIC B, HIRONO J, SATO T, BUCK LB (1999) Combinatorial receptor codes for odors. Cell 96: 713-723

MARTIN C, GERVAIS R, HUGUES E, MESSAOUDI B, RAVEL N (2004) Learning modulation of odor-induced oscillatory responses in the rat olfactory bulb: A correlate of odor recognition? J Neurosci 24: 389-397

MOMBAERTS $\mathrm{P}$, WANG $\mathrm{F}$, DULAC $\mathrm{C}$, CHAO SK, NEMES A, MENDELSOHN M, EDMONDSON J, AXEL R (1996) Visualizing an olfactory sensory map. Cell 87: 675-686

NEVILLE KR, HABERLY LB (2003) Beta and gamma oscillations in the olfactory system of the urethaneanesthetized rat. J Neurophysi 90: 3921-3930

NIKONOV AA, PARKER JM, CAPRIO J (2002) Odorantinduced olfactory receptor neural oscillations and their modulation of olfactory bulbar responses in the channel catfish. J Neurosci 22: 2352-2362

OGAWA Y (1998) Firing properties of olfactory bulb neurons during sniffing in rats. Physiol \& Behav 64: 755-764

ONODA N, MORI K (1980) Depth distribution of temporal firing patterns in olfactory bulb related to air-intake cycles. J Neurophysiol 44: 29-39

PAGER J (1985) Respiration and olfactory bulb unit 
activity in the unrestrained rat: statements and reappraisals. Behav Brain Res 16: 81-94

RAVEL N, PAGER J (1990) Respiratory patterning of the rat olfactory bulb unit activity: nasal versus tracheal breathing. Neurosci Lett 115: 213-218

RAVEL N, CAILLE D, PAGER J (1987) A centrifugal respiratory modulation of olfactory bulb unit activity: a study on acute rat preparation. Exp Brain Res 65: 623-628

RUBIN BD, KATZ LC (1999) Optical imaging of odorant representations in the mammalian olfactory bulb. Neuron 23: 499-511

SINGER W (1999) Neuronal synchrony: a versatile code for the definition of relations? Neuron 24: 49-25

SOBEL EC, TANK DW (1993) Timing of odor stimulation does not alter patterning of olfactory bulb unit activity in freely breathing rats. J Neurophysiol 69: 1331-1337

ZHENG L.M., RAVEL N., JOURDAN F. (1987) Topography of centrifugal acetylcholinesterasepositive fibres in the olfactory bulb of the rat: evidence for original projections in atypical glomeruli. Neurosci 23: $1083-1093$ 